\title{
ON THE SMOOTHED TOEPLITZ EXTENSIONS AND $K$-THEORY
}

\author{
RONGHUI JI
}

(Communicated by John B. Conway)

\begin{abstract}
We construct a smoothed version of the Toeplitz extension for a $C^{*}$-dynamical system $(A, \alpha, \mathbf{R})$. It defines a Thom class $[\tau]$ in $K K^{1}(A$, $\left.A \times{ }_{\alpha} \mathbf{R}\right)$. The Wiener-Hopf-Rieffel extension for $(A, \alpha, \mathbf{R})$ is shown to be a smoothed Toeplitz extension which is the $K K$-inverse of $[\tau]$.
\end{abstract}

\section{INTRODUCTION}

In [2], A. Connes proved an analogue for crossed products of the Thom isomorphism. Since the original proof of Connes' Thom isomorphism theorem is rather complicated, many others have tried to produce proofs from a different point of view [8, 12, 14]. In [9], a smoothed version of the Toeplitz extension for an almost periodic flow on an $n$-dimensional torus is studied and is used to compute the index of Fredholm Toeplitz operators on the flow [4, 10]. It turns out that the smoothed Toeplitz extension for an almost periodic flow provides an easy way to compute the Thom isomorphism. The purpose of the present paper is to generalize the construction of the smoothed Toeplitz extensions made in [9] to any $C^{*}$-dynamical system $(A, \alpha, \mathbf{R})$. As the authors of [9] pointed out, the smoothed Toeplitz extension ought to be dual to the Wiener-Hopf extension constructed by M. Rieffel [14], in the sense of $K K$ theory [1]. We will show in this paper that this is actually true for any $C^{*}$ dynamical system $(A, \alpha, \mathbf{R})$. Therefore, computing the boundary maps of the six term exact sequence of $K$-groups associated with the smoothed Toeplitz extension will yield Connes' Thom isomorphism. In the special case when $\mathbf{R}$ acts on the $n$-dimensional torus as an almost periodic flow, such a study has been accomplished in [9]. It is suggested from the work of $[7,9,11]$ and some unpublished work of $\mathrm{W}$. Arveson that smoothed Toeplitz extensions will become a useful ingredient in the Toeplitz index theory of a $C^{*}$-dynamical system $(A, \alpha, \mathbf{R})$. The standard references for $C^{*}$-dynamical systems and $K$ -

Received by the editors January 1988 and, in revised form, June 19, 1989.

1980 Mathematics Subject Classification (1985 Revision). Primary 19K33, 46L55, 46L80, 47B35.

Key words and phrases. Toeplitz extension, $C^{*}$-algebras, Thom class, $K$-theory, $C^{*}$-dynamical systems.

The author was supported in part by a grant from the National Science Foundation. 
theory are [13] and [1], respectively. This paper shares the same spirit as that in [9].

\section{SMoothed Toeplitz EXTENSIONS}

A $C^{*}$-dynamical system $(A, \alpha, \mathbf{R})$ is a separable $C^{*}$-algebra $A$ and a strong continuous homomorphism $\alpha$ of $\mathbf{R}$ into the group of *-automorphisms of $A$. Without loss of generality we may assume $A$ has a unit which is preserved by $\alpha$. We represent $A$ faithfully on a Hilbert space $H$ and identify $A$ with its image. Let $L^{2}(\mathbf{R}, H)$ be the Hilbert space of $L^{2}$ functions with values in $H$. Then the crossed product $C^{*}$-algebra $A \times{ }_{\alpha} \mathbf{R}$ is faithfully represented on $L^{2}(\mathbf{R}, H)$ via the regular representation [13]. Let $L^{1}(\mathbf{R}, A)$ be the Banach algebra of $L^{1}$ functions with values in $A$ with the twisted convolution as the product:

$$
\varphi *_{r} \psi(t)=\int \varphi(\eta) \alpha_{\eta}(\psi(t-\eta)) d \eta
$$

Then $L^{1}(\mathbf{R}, A)$ can be identified with a dense subalgebra of $A \times{ }_{\alpha} \mathbf{R}$. The regular representation of $A \times_{c} \mathbf{R}$ is defined by

$$
\varphi(f)(t)=\int \alpha_{-t}(\varphi(\eta))(f(t-\eta)) d \eta,
$$

where $\varphi$ is in $L^{1}(\mathbf{R}, A)$, and $f$ is in $L^{2}(\mathbf{R}, H)$.

Let $\hat{\mathbf{R}}$ be the dual group of $\mathbf{R}$. The Fourier transform $F$ from $L^{2}(\mathbf{R})$ onto $L^{2}(\hat{\mathbf{R}})$ extends naturally to an isometry from $L^{2}(\mathbf{R}, H)$ onto $L^{2}(\hat{\mathbf{R}}, H)$. Since $C^{*}(\mathbf{R}) \cong C_{0}(\hat{\mathbf{R}})$ is embedded in $F\left(A \times{ }_{\alpha} \mathbf{R}\right) F^{-1}$ as a $C^{*}$-sub-algebra, a function $\hat{\varphi}$ in $C_{0}(\hat{\mathbf{R}})$ acts on $L^{2}(\hat{\mathbf{R}}, H)$ by multiplication. Let $P_{\varepsilon}$ be a continuous function on $\hat{\mathbf{R}}$, satisfying $P_{\varepsilon}(\xi)=\left\{\begin{array}{ll}0 & \xi \leq 0, \\ 1 & \xi>\varepsilon\end{array}\right.$, and $P_{\varepsilon}(\xi) \geq 0$, for a fixed $\varepsilon>0$. Then $P_{\varepsilon}$ is in the multiplier algebra of $F\left(A \times{ }_{1} \mathbf{R}\right) F^{-1}$. Let us denote $F\left(A \times{ }_{\alpha} \mathbf{R}\right) F^{-1}$ by $\mathscr{C}_{\infty}$.

Lemma 1. Let $\hat{P}_{\varepsilon}$ be the operator $F^{-1} P_{\varepsilon} F$ on $L^{2}(\mathbf{R}, H)$ and let $a$ be in $A$. Then the commutator $\left[\hat{P}_{\varepsilon}, a\right]$ is in $A \times{ }_{1 r} \mathbf{R}$.

Proof. Let $F_{\varepsilon}=2 \hat{P}_{\varepsilon}-1$. Then $F_{\varepsilon}$ is a Thom operator on $A \times{ }_{\alpha} \mathbf{R}[1,8]$. Hence $\left[F_{\varepsilon}, a\right]$ is in $A \times{ }_{\alpha} \mathbf{R}$. Consequently $\left[\hat{P}_{\varepsilon}, a\right]$ is also in $A \times{ }_{\alpha} \mathbf{R}$.

Let $\hat{\mathscr{T}}_{\infty}$ be the $C^{*}$-algebra on $L^{2}(\hat{\mathbf{R}}, H)$ generated by $\mathscr{C}_{\infty}$ and $P_{\varepsilon} F a F^{-1} P_{\varepsilon}$, where $a$ is in $A . \mathscr{C}_{\infty}$ is an ideal of $\hat{\mathscr{T}}_{\infty}$. By Lemma $1,\left[P_{\varepsilon}, F a F^{-1}\right]$ is in $\mathscr{C}_{\infty}$, so that the map $\gamma: A \rightarrow \hat{\mathscr{T}}_{\infty} / \mathscr{C}_{\infty}$ defined by $\gamma(a)=P_{\varepsilon} F a F^{-1} P_{\varepsilon}+\mathscr{C}_{\infty}$ is a surjective $*$-homomorphism. We will show that $\gamma$ is actually a $*$-isomorphism. Note that the algebra $\hat{\mathscr{T}}_{\infty}$ does not depend on the $\varepsilon$ chosen. In fact, one can replace $P_{\varepsilon}$ by any real valued continuous function $h$ on $\hat{\mathbf{R}}$ satisfying $\lim _{\xi \rightarrow-\infty} h(\xi)=0$ and $\lim _{\xi \rightarrow \infty} h(\xi)=1$ without changing the algebra. This is due to the fact that $P_{\varepsilon}-h$ is in $\mathscr{C}_{\infty}$. 
Lemma 2. If $\hat{f}$ be the Fourier transform of $f$ in $L^{2}(\mathbf{R}, H), \varphi$ be in $L^{1}(\mathbf{R}, A)$ and let $d \zeta$ denote the Plancherel measure of $\mathbf{R}$. Then

$$
F \varphi F^{-1}(\hat{f})(\xi)=\int e^{-i \zeta \xi} \alpha_{-\zeta}\left(\hat{\varphi}_{c k}(\xi)\right)(f(\zeta)) d \zeta
$$

where $\hat{\varphi}_{c \alpha}(\xi)=\int e^{-i t \xi} \alpha_{-t}(\varphi(t)) d t$ converges in norm, and $\lim _{\xi \rightarrow \pm \infty} \hat{\varphi}_{\alpha}(\xi)=0$. Proof.

$$
\begin{aligned}
F \varphi F^{-1}(\hat{f})(\xi) & =\int e^{-i t \xi} \varphi(f)(t) d t \\
& =\int e^{-i t \xi} \int \alpha_{-t}(\varphi(\eta))(f(t-\eta)) d \eta d t \\
& =\int e^{-i t \xi} \int \alpha_{-t}(\varphi(t-\zeta))(f(\zeta)) d \zeta d t \\
& =\int e^{-i \zeta \xi}\left\{\int e^{-i s \xi} \alpha_{-s-\zeta}(\varphi(s)) d s\right\}(f(\zeta)) d \zeta \\
& =\int e^{-i \zeta \xi} \alpha_{-\zeta}\left(\hat{\varphi}_{\alpha}(\xi)\right)(f(\zeta)) d \zeta
\end{aligned}
$$

The statement that $\lim _{\xi \rightarrow \pm \infty} \hat{\varphi}(\xi)=0$ follows from the fact that $\varphi$ is in $L^{1}(\mathbf{R}, A)$.

Lemma 3. For $a \neq 0, P_{\varepsilon} F a F^{-1} P_{\varepsilon}$ is not in $\mathscr{C}_{\infty}$.

Proof. Since $\left[P_{\varepsilon}, F a F^{-1}\right]$ and $P_{\varepsilon}^{2}-P_{\varepsilon}$ are in $\mathscr{C}_{\infty}$, we need only to show that $P_{\varepsilon} F a F^{-1}$ is not in $\mathscr{C}_{\infty}$. Let $f$ be in $L^{2}(\mathbf{R}, H), \varphi$ be in $L^{1}(\mathbf{R}, A)$, and $a$ in $A$. We have

$$
P_{\varepsilon} F a F^{-1}(\hat{f})(\xi)-F \varphi F^{-1}(\hat{f})(\xi)=\int e^{-i t \xi} \alpha_{-t}\left(P_{\varepsilon}(\xi) a-\hat{\varphi}_{c k}(\xi)\right)(f(t)) d t .
$$

Since $\lim _{\xi \rightarrow \pm \infty} \hat{\varphi}_{x}(\xi)=0$, it is sufficient to prove that for any compactly supported continuous function $\psi$ from $\hat{\mathbf{R}}$ into $A$, there exists an $f$ in $L^{2}(\mathbf{R}, H)$ such that $\|f\|_{2}=1$ and $\left\|\int e^{-i t \xi} \alpha_{-t}\left(P_{\varepsilon}(\xi) a-\psi(\xi)\right)(f(t)) d t\right\|_{2}^{2} \geq c\|a\|^{2}$ for some $c>0$. In fact, if $\psi(\xi)=0$ for all $\xi \geq N \geq \varepsilon$, then for such $\xi$

$$
\int e^{-i t \xi} \alpha_{-t}\left(P_{\varepsilon}(\xi) a-\psi(\xi)\right)(f(t)) d t=\int e^{-i t \xi} \alpha_{-t}(a)(f(t)) d t .
$$

Since $a$ is nonzero, there exists a vector $v$ in $H$ such that $\|a(v)\|>\frac{1}{2}\|a\|$. Moreover, there exists a $\delta>0$, so that for $N \leq \xi \leq N+1$, and for $0<t<\delta$, $\left|e^{-i t \xi}-1\right|<1 / 8$ and $\left\|\alpha_{-t}(a)-a\right\|<1 / 8\|a\|$. 
Let $f(t)=1 / \delta \chi_{[0, \delta]} \otimes v$. We have

$$
\begin{aligned}
& \left\|\int e^{-i t \xi} \alpha_{-t}\left(P_{\varepsilon}(\xi) a-\psi(\xi)\right)(f(t)) d t\right\|_{2}^{2} \\
& \quad \geq \int_{N}^{N+1}\left(\left\|\int e^{-i t \xi} \alpha_{-t}\left(P_{\varepsilon}(\xi) a-\psi(\xi)\right)(f(t)) d t\right\|^{2}\right) d \xi \\
& \quad=\int_{N}^{N+1}\left\|\int e^{-i t \xi} \alpha_{-t}(a)(f(t)) d t\right\|^{2} d \xi \\
& \quad=\frac{1}{\delta}\left\{\int_{N}^{N+1}\left\|\int_{0}^{\delta} e^{-i t \xi} \alpha_{-t}(a)(v) d t\right\|^{2} d \xi\right\} \\
& \quad \geq \frac{1}{\delta}\left\{\int _ { N } ^ { N + 1 } \left(\left\|\int_{0}^{\delta} a(v) d t\right\|-\left\|\int_{0}^{\delta}\left(e^{-i t \xi}-1\right) a(v) d t\right\|\right.\right. \\
& \quad \geq\left\{\int_{N}^{N+1}\left(\frac{1}{2}\|a\|-\frac{1}{8}\|a\|-\frac{1}{8}\|a\|\right)^{2} d \xi\right\}=\frac{1}{16 \sqrt{2 \pi}}\|a\|^{2} .
\end{aligned}
$$

This proves the lemma.

Theorem 1. With the notation above, we have an exact sequence of $C^{*}$-algebras $0 \rightarrow \mathscr{C}_{\infty} \stackrel{i}{\rightarrow} \hat{\mathscr{T}}_{\infty} \stackrel{q}{\rightarrow} A \rightarrow 0$, where $i$ is the inclusion, and $q$ is the symbol map defined by $q\left(P_{\varepsilon} F a F^{-1} P_{\varepsilon}+\mathscr{C}_{\infty}\right)=a$, for all $a$ in $A$.

Proof. All we have to show is that $\gamma$ defined earlier is injective. In fact, from Lemma $3, \gamma(a)$ is not in $\mathscr{C}_{\infty}$ for all $a$ in $A$. Hence, $\gamma(a)$ is not zero in $\hat{\mathscr{T}} / \mathscr{C}_{\infty}$.

Remark. As we indicated before, the purposes of adding $\mathscr{C}_{\infty}$ into $\hat{\mathscr{T}}_{\infty}$ are both to eliminate the dependence of $\varepsilon$ chosen and to gain a better control of the ideal. However, if one does not wish to do so, one may construct an $\varepsilon$ dependent smoothed Toeplitz extension similar to the one we constructed above. Let $\mathscr{T}_{\varepsilon}$ be the $C^{*}$-algebra generated by the operators $\left\{\hat{P}_{\varepsilon} a \hat{P}_{\varepsilon}, \hat{P}_{\varepsilon}^{2}-\hat{P}_{\varepsilon} \mid a \in A\right\}$ on $L^{2}(\mathbf{R}, H)$. Let $\mathscr{C}_{\varepsilon}$ be the closed $*$-ideal of $\mathscr{T}_{\varepsilon}$ generated by the elements of $\left\{\hat{P}_{\varepsilon} a \hat{P}_{\varepsilon} b \hat{P}_{\varepsilon}-\hat{P}_{\varepsilon} a b \hat{P}_{\varepsilon}, \hat{P}_{\varepsilon}^{2}-\hat{P}_{\varepsilon} \mid a, b \in A\right\}$. Note that $\mathscr{T}_{\varepsilon} \subset \hat{\mathscr{T}}_{\infty}$, and $\mathscr{C}_{\varepsilon} \subset \mathscr{C}_{\infty}$. By Theorem 1 it is easy to deduce that the sequence $0 \rightarrow \mathscr{C}_{\varepsilon} \rightarrow \mathscr{T}_{\varepsilon} \rightarrow A \rightarrow 0$ is exact. Motivated by this observation, one may define the genuine Toeplitz extension as follows. Let

$$
P(\xi)= \begin{cases}1 & \xi \geq 0, \\ 0 & \xi<0,\end{cases}
$$

on $L^{2}(\hat{\mathbf{R}}, H)$, and let $\hat{P}=F^{-1} P F$ on $L^{2}(\mathbf{R}, H)$. Define $\mathscr{T}$ to be the $C^{*}$ algebra generated by the operators of the form $\hat{P} a \hat{P}$ for $a$ in $A$. Let $\mathscr{C}$ be 
the ideal of $\mathscr{T}$ generated by the elements of the form $(\hat{P} a \hat{P})(\hat{P} b \hat{P})-\hat{P} a b \hat{P}$, where $a$ and $b$ are in $A$. In view of Theorem 1, one would expect that there is an exact sequence of $C^{*}$-algebras $0 \rightarrow \mathscr{C} \rightarrow \mathscr{T} \rightarrow A \rightarrow 0$. In many cases this is known to be true, e.g. $[4,5,6]$. But in general, the question seems to be open. We would like to give a proof here which is based on a lemma of [5].

Proposition 1. There is an exact sequence of $C^{*}$-algebras

$$
0 \rightarrow \mathscr{C} \rightarrow \mathscr{T} \rightarrow A \rightarrow 0 .
$$

Proof. There is an obvious surjective $*$-homomorphism $\gamma$ from $A$ into $\mathscr{T} / \mathscr{C}$ defined by $\gamma(a)=\hat{P} a \hat{P}+\mathscr{C}$. We must prove that $\gamma$ is actually injective. Let $a$ be in $A$, and let $T_{\lambda}$ be the translation on $L^{2}(\hat{\mathbf{R}}, H)$ defined by $T_{\lambda}(f)(s)=$ $f(s-\lambda)$. For any $\xi, \eta$ in $L^{2}(\mathbf{R}, H), \lim _{\lambda \rightarrow \infty}\left(\hat{P} a \hat{P} W_{\lambda} \xi, W_{\lambda} \eta\right)=(a(\xi), \eta)$, where $W_{\lambda}=F^{-1} T_{\lambda} F$. In fact, it suffices to show that $\lim _{\lambda \rightarrow \infty}\left\|\hat{P} W_{\lambda} \xi-W_{\lambda} \xi\right\|_{2}$ $=0$. But this follows from the equality $\left\|\hat{P} W_{\lambda} \xi-W_{\lambda} \xi\right\|_{2}=\left\|\chi_{(-\infty,-\lambda)}(F \xi)\right\|_{2}$ by the Fourier transform. If $S$ runs through all sums of products of operators of the form $(\hat{P} a \hat{P})(\hat{P} b \hat{P})-\hat{P} a b \hat{P}$, for $a, b$ in $A$ and $\xi, \eta$ in $L^{2}(\mathbf{R}, H)$, then the above assertion implies that $\lim _{\lambda \rightarrow 0}\left(S W_{\lambda} \xi, W_{\lambda} \eta\right)=0$. Therefore, $\|a\|=\sup \{|(a(\xi), \eta)|:\|\xi\|=\|\eta\|=1\}=\sup \left\{\overline{\lim }_{\lambda \rightarrow \infty}\left|\left((\hat{P} a \hat{P}+S) W_{\lambda} \xi, W_{\lambda} \eta\right)\right|:\right.$ $\|\xi\|=\|\eta\|=1\}=\sup \{|((\hat{P} a \hat{P}+S) \xi, \eta)|:\|\xi\|=\|\eta\|=1\}=\|\hat{P} a \hat{P}+S\|$ for all $S$. Consequently, $\|a\|=\|\hat{P} a \hat{P}+\mathscr{C}\|$, the latter being the norm of the quotient algebra $\mathscr{T} / \mathscr{C}$. This establishes the injectivity of $\gamma$, so the proposition is proved. (cf. [5, Lemma 24.2].)

Remark. In general, there is no direct relationship between the algebras $\mathscr{T}$ and $\hat{\mathscr{T}}_{\infty}$ except that $\mathscr{T}$ is in the weak $*$-closure of $\hat{\mathscr{T}}_{\infty}$. However, in some cases (e.g. $[9,11])$, when one compresses $\hat{\mathscr{T}}_{\infty}$ by $\hat{P}$, one may have $\hat{P} \hat{\mathscr{T}}_{\infty} \hat{P} \subset \mathscr{T}$, and $\hat{P} \hat{\mathscr{C}}_{\infty} \hat{P} \subset \mathscr{C}$. This containment allows one to obtain the $K$-theoretical index (in $K_{0}\left(A \times{ }_{\alpha} \mathbf{R}\right)$ ) of a Fredholm operator in $\mathscr{T}$ relative to the ideal $\mathscr{C}$ by using the smoothed Toeplitz extension $[10,11]$.

\section{THOM CLASSES AND THE WIENER-HOPF EXTENSION}

It was proved in [9] that the smoothed Toeplitz extension for an almost periodic flow on an $n$-torus defines a Thom class in $K K^{1}\left(A, A \times_{\alpha} \mathbf{R}\right)$, where $A=C\left(T^{n}\right)$. The same statement holds in the general case.

Theorem 2. Under the canonical isomorphism of $K K^{1}\left(A, A \times_{a} \mathbf{R}\right)$ with $\operatorname{Ext}\left(A, A \times{ }_{\alpha} \mathbf{R}\right)$ [1], the smoothed Toeplitz extension is the Thom element in $K K^{1}\left(A, A \times{ }_{\alpha} \mathbf{R}\right)$.

Proof. Let $M\left(A \times{ }_{\alpha} \mathbf{R}\right)$ denote the multiplier algebra of $A \times_{\alpha} \mathbf{R}$, and let $Q\left(A \times{ }_{\alpha} \mathbf{R}\right)$ denote the quotient $M\left(A \times{ }_{\alpha} \mathbf{R}\right) / A \times{ }_{\alpha} \mathbf{R}$. Let $\pi$ be the natural projection from $M\left(A \times_{\alpha} \mathbf{R}\right)$ onto $Q\left(A \times_{\alpha} \mathbf{R}\right)$. From the exact sequence $0 \rightarrow \mathscr{C}_{\infty} \rightarrow \hat{\mathscr{T}}_{\infty} \rightarrow A \rightarrow 0$ defined in $\S 2$, we obtain the following exact sequence via the Fourier transform $F: 0 \rightarrow A \times_{k r} \mathbf{R} \rightarrow \mathscr{T}_{\infty} \rightarrow A \rightarrow 0$. 
Hence, one can define a $*$-homomorphism $\tau: A \rightarrow Q\left(A \times_{\alpha} \mathbf{R}\right)$ by $\tau(a)=$ $\pi\left(\hat{P}_{\varepsilon} a \hat{P}_{\varepsilon}\right)$. Since $\mathscr{T}_{\infty}$ is contained in $M\left(A \times{ }_{\alpha} \mathbf{R}\right)$, the extension $0 \rightarrow A \times{ }_{\alpha} \mathbf{R} \rightarrow$ $\mathscr{T}_{\infty} \rightarrow A \rightarrow 0$ is exactly the extension corresponding to the Kasparov $A-A \times{ }_{\alpha} \mathbf{R}$ bimodule $\left(A \times_{\alpha} \mathbf{R}, \pi,\left(2 \hat{P}_{\varepsilon}-1\right)\right)([1,19.3])$ under the canonical isomorphism from $K K^{1}\left(A, A \times{ }_{\alpha} \mathbf{R}\right)$ onto $\operatorname{Ext}\left(A, A \times{ }_{\alpha} \mathbf{R}\right)$. Here $\pi$ is the embedding of $A$ into $M\left(A \times{ }_{\alpha} \mathbf{R}\right)$ defined in $\S 2$ (cf. [1, 15.3 and 19.3] and [9]).

The construction of the smoothed Toeplitz extension depends on the initial representation of the $C^{*}$-algebra $A$. However, it is unique up to isomorphism.

Proposition 2. Let $\pi$ and $\pi^{\prime}$ be faithful non-degenerate representations of $A$ on the Hilbert spaces $H$ and $H^{\prime}$, respectively. Then the smoothed Toeplitz extensions for $(A, \alpha, \mathbf{R})$ constructed using $\pi$ and $\pi^{\prime}$ are canonically isomorphic.

Proof. Since the multiplier algebras $M\left(A \times{ }_{\alpha} \mathbf{R}\right)$ on $L^{2}(\mathbf{R}, H)$ and $M^{\prime}\left(A \times{ }_{\alpha} \mathbf{R}\right)$ on $L^{2}\left(\mathbf{R}, H^{\prime}\right)$ are canonically isomorphic, and the images of the embeddings of $C^{*}(\mathbf{R}) \cong C_{0}(\hat{\mathbf{R}})$ in them are canonically isomorphic, the images of $C^{b}(\hat{\mathbf{R}})$ in these multiplier algebras are also canonically isomorphic. One can easily verify that the Toeplitz algebras constructed from the two representations are canonically isomorphic under the isomorphism of the multiplier algebras above. Therefore we have a diagram

$$
\begin{aligned}
& 0 \rightarrow \mathscr{C}_{\infty} \rightarrow \hat{\mathscr{T}}_{\infty} \rightarrow A \rightarrow 0 \\
& 0 \rightarrow \hat{\mathscr{C}}_{\infty}^{\prime \cong} \rightarrow \mathscr{\mathscr { T }}_{\infty}^{\prime} \rightarrow A \cong \rightarrow
\end{aligned}
$$

The commutativity of this diagram is easily checked using the above observation. This proves the proposition.

We can now speak of the smoothed Toeplitz extension $0 \rightarrow A \times{ }_{\alpha} \mathbf{R} \rightarrow \mathscr{T}_{\infty} \rightarrow$ $A \rightarrow 0$ for the dynamical system $(A, \alpha, \mathbf{R})$ without specifying the faithful representation of $A$.

Let $(A, \alpha, \mathbf{R})$ and $\left(A \times_{\alpha} \mathbf{R}, \beta, \mathbf{R}\right)$ be $C^{*}$-dynamical systems. The latter induces an action $\tilde{\beta}$ of $\mathbf{R}$ on $M\left(A \times{ }_{\alpha} \mathbf{R}\right)$. We assume that $A$ and $C^{*}(\mathbf{R}) \cong$ $C_{0}(\hat{\mathbf{R}})$ are invariant under $\tilde{\beta}$, and that $\tilde{\beta}$ preserves the natural orientation of $\hat{\mathbf{R}}$.

Lemma 4. Under above hypothesis the smoothed Toeplitz algebra $\mathscr{T}_{\infty}$ of the $C^{*}$ dynamical system $(A, \alpha, \mathbf{R})$ is invariant under $\tilde{\beta}$. Moreover, one has an exact sequence of $C^{*}$-algebras $0 \rightarrow\left(A \times{ }_{\alpha} \mathbf{R}\right) \times{ }_{\beta} \mathbf{R} \rightarrow \mathscr{T}_{\infty} \times{ }_{\dot{\beta}} \mathbf{R} \rightarrow A \times \times_{\bar{\beta}} \mathbf{R} \rightarrow 0$.

Proof. Recall that the $C^{*}$-algebra $\mathscr{T}_{\infty}$ is generated by $A \times{ }_{\alpha} \mathbf{R}$ and $\hat{P}_{\varepsilon} a \hat{P}_{\varepsilon}$. Since $A$ and $C^{*}(\mathbf{R}) \cong C_{0}(\hat{\mathbf{R}})$ are invariant under $\tilde{\beta}$, and $\tilde{\beta}$ preserves the orientation of $\hat{\mathbf{R}}$, we see that $\tilde{\beta}_{t}\left(\hat{P}_{\varepsilon}\right)$ differs from $\hat{P}_{\varepsilon}$ by an element of $A \times{ }_{1} \mathbf{R}$. Thus the first statement follows. The second statement follows from [2, Lemma 1]. 
In Lemma 4 , if $\tilde{\beta}$ acts on $C^{*}(\mathbf{R}) \cong C_{0}(\hat{\mathbf{R}})$ by translation, $\tilde{\beta}_{\xi}(f)(\eta)=f(\eta+\xi)$, then there is an induced action $\tilde{\alpha}$ of $\alpha$ on $M\left(A \times_{\bar{\beta}} \mathbf{R}\right)$, so that $\tilde{\alpha}_{t}(a)=\alpha_{t}(a)$ and $\tilde{\alpha}_{t}(g)(s)=g(s-t)$, where $g$ is in $C_{0}(\hat{\mathbf{R}})$.

Lemma 5. Let $\alpha, \tilde{\alpha}, \beta$, and $\tilde{\beta}$ be as above. Then the exact sequence in Lemma 4 is naturally isomorphic to the smoothed Toeplitz extension of the $C^{*}$-dynamical system $\left(A \times_{\grave{\beta}} \tilde{\alpha}, \mathbf{R}\right)$.

Proof. Let $\mathbf{R}^{\prime}$ be the group $\mathbf{R}$ corresponding to the $\beta$ action. Since the action of $\tilde{\beta}$ on $C^{*}(\mathbf{R})$ is the dual action, $\left(A \times_{\alpha} \mathbf{R}\right) \times_{\beta} \mathbf{R}^{\prime}$ is naturally isomorphic to $\left(A \times_{\bar{\beta}} \mathbf{R}^{\prime}\right) \times_{i \dot{r}} \mathbf{R}$. The isomorphism is implemented by a unitary $U$ from $L^{2}\left(\mathbf{R} \times \mathbf{R}^{\prime}, H\right)$ onto $L^{2}\left(\mathbf{R}^{\prime} \times \mathbf{R}, H\right)$, which is defined by $U(f)(t, \xi)=f(\xi, t)$. Recall that $A$ is faithfully represented on $H$, and the algebras $\left(A \times{ }_{\alpha} \mathbf{R}\right) \times{ }_{\beta} \mathbf{R}^{\prime}$ and $\left(A \times_{\bar{\beta}} \mathbf{R}^{\prime}\right) \times_{i \bar{\alpha}} \mathbf{R}$ are represented by the regular representation on $L^{2}\left(\mathbf{R}^{\prime} \times\right.$ $\mathbf{R}, H)$ and on $L^{2}\left(\mathbf{R} \times \mathbf{R}^{\prime}, H\right)$, respectively. Let $\mathscr{T}_{\infty}$ be the smoothed Toeplitz algebra of $(A, \alpha, \mathbf{R})$, which is defined on $L^{2}(\mathbf{R}, H)$. Then $\mathscr{T}_{\infty} \times_{\dot{\beta}} \mathbf{R}^{\prime}$ is represented on $L^{2}\left(\mathbf{R} \times \mathbf{R}^{\prime}, H\right)$ via the regular representation. Let $\Omega_{\infty}$ be the smoothed Toeplitz extension of the dynamical system $\left(A \times_{\bar{\beta}} \mathbf{R}^{\prime}, \tilde{\alpha}, \mathbf{R}\right)$, which is defined on $L^{2}\left(\mathbf{R} \times \mathbf{R}^{\prime}, H\right)$. We will prove that the $C^{*}$-algebra $\mathscr{T}_{\infty} \times_{\bar{\beta}} \mathbf{R}^{\prime}$ is naturally isomorphic to $\Omega_{\infty}$ via conjugation by the unitary $U$. It is clear that $\Omega_{\infty}$ is generated by $\left(A \times_{\bar{\beta}} \mathbf{R}^{\prime}\right) \times_{\bar{\alpha}} \mathbf{R}$ and $\hat{P}_{\varepsilon} \varphi \hat{P}_{\varepsilon}$ for $\varphi$ in $A \times_{\dot{\beta}} \mathbf{R}^{\prime}$. Moreover, $U^{*}\left(\mathscr{T}_{\infty} \times_{\bar{\beta}} \mathbf{R}^{\prime}\right) U$ is generated by $U^{*}\left[\left(A \times{ }_{\alpha} \mathbf{R}\right) \times_{\dot{\beta}} \mathbf{R}^{\prime}\right] U=\left(A \times_{\bar{\beta}} \mathbf{R}^{\prime}\right) \times_{\bar{\alpha}} \mathbf{R}$ and $\hat{P}_{\varepsilon} \psi \hat{P}_{\varepsilon}$, where $\psi$ runs through all compactly supported continuous functions from $\mathbf{R}^{\prime}$ into $A$. These are represented on $L^{2}\left(\mathbf{R}^{\prime} \times \mathbf{R}, H\right)$ by the regular representation $\varphi(f)(\xi, t)=\tilde{\beta}_{-\xi}\left(\tilde{\alpha}_{-t}(\varphi)(\xi)\right)(f(\xi, t))$. From this it is easy to check that the last claim is true. This proves the lemma.

Corollary. Let $(A, \alpha, \mathbf{R})$ be a dynamical system. Then the Wiener-Hopf extension of [14] is a smoothed Toeplitz extension. Hence, it defines a Thom element in $K K^{1}\left(A \times{ }_{\alpha} \mathbf{R}, A\right)$, which is the $K K$-inverse of the smoothed Toeplitz extension of $(A, \alpha, \mathbf{R})$.

Proof. Let $\hat{\alpha}$ be the dual action of $\alpha$. Let $0 \rightarrow A \times \times_{\hat{\alpha}} \mathbf{R} \cong A \otimes C_{0}(\hat{\mathbf{R}}) \rightarrow \tilde{\mathscr{T}}_{\infty} \rightarrow$ $A \rightarrow 0$ be the smoothed Toeplitz extension of the dynamical system $(A, \hat{\alpha}, \hat{\mathbf{R}})$. Note $\hat{\alpha}$ acts on $A$ trivially. We claim that the smoothed Toeplitz extension

$$
0 \rightarrow\left(A \times_{i \alpha} \hat{\mathbf{R}}\right) \times{ }_{\alpha} \mathbf{R} \cong A \otimes \mathscr{K} \rightarrow \tilde{\mathscr{T}}_{\infty} \times{ }_{i x} \mathbf{R} \rightarrow A \times{ }_{\alpha} \mathbf{R} \rightarrow 0
$$

is exactly the Wiener-Hopf extension of [14], where $\tilde{\alpha}$ is the induced action of $\alpha$ on $M\left(A \times_{\hat{\alpha}} \hat{\mathbf{R}}\right)$. All we need to show is that $\tilde{\mathscr{T}}_{\infty} \times_{i \dot{\alpha}} \mathbf{R} \cong C A \times_{i}, \mathbf{R}$, where $C A=C_{0}(\mathbf{R} \cup\{+\infty\}) \otimes A$, and where $\gamma$ acts on $C A$ by $\gamma_{t}(f \otimes a)=f_{t} \otimes a$ with $f_{t}(s)=f(s-t)$. In fact, since $\hat{\alpha}$ acts on $A$ trivially, $\tilde{\mathscr{T}}_{\infty}$ is canonically isomorphic to $C A$, and the action of $\alpha$ on $\tilde{\mathscr{T}}_{\infty}$ gives the action $\gamma$ on $C A$ under the isomorphism. This proves the claim and the corollary follows. 
Added in proof. The author recently learned that Theorem 1 of this paper has been independently obtained by W. Arveson.

\section{ACKNOWLEDGMENT}

The author would like to thank Ronald G. Douglas and Jerry Kaminker for many helpful conversations and comments.

\section{REFERENCES}

1. B. Blackadar, K-Theory for operator algebras, M.S.R.I. Publications 5, Springer-Verlag (1986).

2. A. Connes, An analogue of the Thom isomorphism for crossed products of a $C^{*}$-algebra by an action of $\mathbf{R}$, Adv. in Math. 39 (1981), 31-55.

3.,$- C^{*}$-algebres et geometrie differentielle, C. R. Acad. Sc. Paris, t. 290 (31 mars 1980) Serie A, 599-604.

4. L. Coburn, R. G. Douglas, D. G. Schaeffer and I. M. Singer, On $C^{*}$-algebras of operators on a half-space, II: index theory, Publ. I.H.E.S. 40 (1971), 69-79.

5. R. Curto, P. Muhly and J. Xia, Toeplitz operators on flows, preprint.

6. R. G. Douglas, On the $C^{*}$-algebra of a one-parameter semigroup of isometries, Acta Math. 128 (1972), 143-151.

7. R. G. Douglas, S. Hurder, and J. Kaminker, The longitudinal cyclic cocycle and the index of Toeplitz operators, preprint.

8. T. Fack and G. Skandalis, Connes' analogue of the Thom isomorphism for the Kasparov groups, Invent. Math. 64 (1981), 7-14.

9. R. Ji and J. Kaminker, The K-theory of Toeplitz extensions, J. Operator Theory 19 (1988), 247-354.

10. R. Ji and J. Xia, On the classification of commutator ideals, J. Funct. Anal. 78 (1988), 208-232.

11. R. Ji, Toeplitz operators on non-commutative tori and their real valued index, (to appear in the Proceedings of the AMS Summer Institute in Operator Theory/Operator Algebras, held in Durham, New Hampshire).

12. W. Paschke, On the mapping torus of an automorphism, Proc. Amer. Math. Soc. 88 (1983), 481-485.

13. G. Pederson, $C^{*}$-algebras and their automorphism groups, Academic Press, New York, 1979.

14. M. Rieffel, Connes' analogue for crossed products of the Thom isomorphism, in Operator Algebras and $K$-Theory, Contemp. Math. (R. Douglas and C. Schochet, eds.), 10, Amer. Math. Soc., Providence, RI, 1982.

Department of Mathematical Sciences, Indiana University-Purdue University at INDIANAPOLIS, 1125 EAST 38Th STREET, INDIANAPOLIS, INDIANA 46205

Current address: Department of Mathematics, University of California, Berkeley, California 94720 